\title{
Antitumor Memory T-Cells Become Functionally Mature from 30 to 100 days in a Mouse Model of Neoplasia
}

\author{
YANHUA GAO ${ }^{1}$, MAMDOUHA A. BARMADA ${ }^{2}$ and IRA BERGMAN $1,3,4$ \\ Departments of ${ }^{1}$ Pediatrics, ${ }^{3}$ Neurology, and ${ }^{4}$ Immunology, Children's Hospital of Pittsburgh, \\ University of Pittsburgh School of Medicine, Pittsburgh, PA, U.S.A.; \\ ${ }^{2}$ MMA-NMD Lab, Department of Pathology and Laboratory Medicine, \\ American University of Beirut Medical Center, American University Hospital, Beirut, Lebanon
}

\begin{abstract}
Background: Late metastases develop from cancer of the breast, prostate, lung, kidney and malignant melanomas. Memory T-cells have excellent potential to prevent this devastating development in the same way that they routinely prevent emergence of latent viruses. Material and Methods: A peritoneal tumor mouse model of viral oncotherapy was used to generate therapeutic antitumor memory T-cells. Functional in vivo and in vitro assays were used to study the temporal evolution of their anticancer effects. Results: Highly therapeutic antitumor memory was generated by viral oncolytic immunotherapy 30 days after treatment and matured to maximal potency at 100 days. Maturation was not uniform across different measures. Conclusion: The results provide guidelines for developing a viral oncolytic vaccine strategy to generate antitumor memory T-cells that can eliminate small nests of metastatic cancer cells in sanctuary sites and prevent emergence of tumors from dormant cancer cell collections. The results are relevant to any immunization strategy designed to generate antitumor memory T-cells.
\end{abstract}

Memory T-cells are necessary for protection from some viruses, bacteria and fungi and most especially from herpesviruses $(1,2)$. Exposure to these agents results in proliferation and differentiation of a small number of $\mathrm{CD} 4^{+}$and $\mathrm{CD}^{+} \mathrm{T}$-cells that recognize foreign antigens presented by host major histocompatibility complex class 1 and 2 proteins (3). When an initial infection is eliminated or suppressed, the host retains a larger number of memory T-cells that recognize the specific foreign antigens. These memory T-cells have improved

Correspondence to: Ira Bergman, Children's Hospital of Pittsburgh of UPMC One Children's Hospital Drive 4401 Penn Avenue Pittsburgh, PA 15224, U.S.A. Tel: +1 4126927176, Fax: +1 4126927303, e-mail: ira.bergman@chp.edu

Key Words: Memory T-cells, immunotherapy, oncotherapy, vesicular stomatitis virus. capacity to combat re-infection or recurrence of infection, including more sensitive, faster and greater proliferation, cytokine production and cell killing with corresponding transcriptional, epigenetic and metabolic modifications (4-8). Work with mouse and human viral infection has demonstrated that memory T-cell maturation continues from day 30 to day 90 after exposure to viral antigens (9-13).

Targeting T-cells in order to eliminate neoplasms has biological plausibility because T-cells are: cytolytic and recruit inflammatory cells which are cytolytic; can produce autoimmune diseases which eliminate specific cell populations including beta cells of the pancreas, thyroxine-producing cells of the thyroid gland and hypocretin-secreting cells of the hypothalamus; are critical mediators of transplant rejection and have already been adapted as chimeric antigen receptor Tcells to inhibit and eliminate some types of hematological cancer (4, 14-17).

We have previously shown in a mouse model of implanted peritoneal tumor that treatment with a targeted replicating recombinant vesicular stomatitis virus (rrVSV) generates highly potent therapeutic antitumor memory T-cells that are resistant to inhibition by suppressor cells (18-21). We now used functional in vivo and in vitro T-cell assays to determine the time course of antitumor T-cell maturation. This knowledge would help provide guidelines for developing a viral oncolytic vaccine strategy to generate antitumor memory T-cells that prevent emergence of dormant metastases from cancers of the breast, prostate, lung, kidney and malignant melanomas (22-24).

\section{Materials and Methods}

Cells, antibodies, chemicals and animals. D2F2/E2 cells, a mouse mammary tumor line that has been stably transfected with a vector expressing the human epidermal growth factor receptor 2 (HER2/neu) gene and its parent tumor cell line, D2F2, were a generous gift from Dr. Wei-Zen Wei (Karmanos Cancer Institute, Wayne State University, Detroit, MI, USA) in 2001. Early passage cells were frozen and periodically thawed for experimental use or 
restocking. Thawed cells were used experimentally for 5-10 passages. Mycoplasma testing was negative using the Impact III PCR profile from IDEXX (RADIL, Columbia, MO, USA).

Antibody to cytotoxic T-lymphocyte-associated protein 4 (CTLA4) (9H10) was obtained commercially (BioXcell Fermentation/Purification Services, West Lebanon, NH, USA). AntiCD8 (2.43 antibody) (26) and anti-CD4 (GK1.5 antibody) (27) ascites were prepared from hybridomas obtained from the American Type Culture Collection (Rockville, MD, USA).

Mice were 8 to 20 weeks of age and weighed 20-25 g. Thy 1.2 $\mathrm{BALB} / \mathrm{c}$ were obtained from Taconic (Hudson, NY). A mating pair of Thy 1.1 BALB/c were purchased from the Jackson Laboratory (Strain name: CBy.PL(B6)-Thy ${ }^{a / S c r J}$, Stock Number: 005443) and bred on site. Animal studies were approved by the institutional Animal Research and Care Committee (approval number: IS00005591).

Preparation of $r r V S V$. A replicating virus was created from vector components as previously described (25) and had the following properties: Preferential infection of cells expressing human HER2/neu, expression of mouse Granulocyte-macrophage colony-stimulating factor, and expression of enhanced green fluorescent protein. Construction used vectors generously supplied by Dr. John K. Rose (Department of Pathology, Yale University, New Haven, CT, USA), Dr. Irvin S. Y. Chen (Department of Microbiology, Immunology, Molecular Genetics, University of California, Los Angeles, CA, USA) and Genentech Inc., South San Francisco, CA, USA).

Viral immuno-oncotherapy. Female BALB/c Thy 1.2 mice, 8 to 20 weeks of age, were implanted intraperitoneally (i.p.) with $2 \times 10^{6}$ D2F2/E2 cells in $300 \mu$ l phosphate-buffered saline (PBS). On day 3 they were treated with $1 \times 10^{8} \mathrm{rrVSV}$ i.p., on day 4 with $200 \mu \mathrm{g}$ anti-CTLA4 and on day 5 with $\sim 100 \mathrm{mg} / \mathrm{kg}$ cyclophosphamide (CTX). The animals were considered cured if they survived for 100 days after tumor cell inoculation. Animals sacrificed at earlier times for timed experiments were visually inspected to ensure that there was no tumor in the peritoneum.

In vivo depletion of T-cells. Total body $\mathrm{CD} 8+\mathrm{T}$-cells were depleted using the 2.43 antibody and CD4+ T-cells were depleted using the GK1.5 antibody as previously described (26).

Cell collection. Memory cells were obtained from spleens of cured animals. Animals were sacrificed prior to cell harvesting. Spleens were harvested, minced and ground through a $70 \mu \mathrm{M}$ nylon cell strainer (BD Falcon, Franklin Lakes, NJ, USA). Red blood cells (RBC) were removed using RBC lysis buffer (Alfa Aesar, Haverhill, MA, USA). Peritoneal washings were performed by injecting $10 \mathrm{ml}$ of sterile PBS into the peritoneum through a 16-gauge needle which was left in place. Five minutes later all the fluid that could be aspirated easily into the syringe was collected. Usually $9.0 \mathrm{ml}$ was collected. All cells were washed twice with PBS and re-suspended in PBS. Total T-cells, CD4+ T-cells and CD8+ T-cells were isolated by positive or negative selection using an autoMACS ${ }^{\mathrm{TM}}$ separator and appropriate antibody microbeads: CD90 (Thy1.2, \#130-049-101), CD4 (L3T4, \#130-049201), and CD8a (Ly-2, \#130-049-401), according to the manufacturer's instructions (Miltenyi Biotec, Auburn, CA, USA).

Adoptive therapy. Peritoneal tumors were established in host animals and treated 3 days later by i.p. adoptive transfer of splenocytes from donor naïve or treated animals. In 1:1 transfers, cells from animals were transferred to the same number of hosts. In 1:2 transfers, cells from animals were transferred to twice as many hosts. As previously described, host animals were pre-treated with CTX one day before transfer of memory cells (18). Animals were assessed three times per week for ascites, abdominal nodules and signs of poor health such as low activity, poor grooming, rough coat, hunched posture and dehydration, and sacrificed if they developed any of these signs. In one set of experiments, carboxyfluorescein succinimidyl ester (CFSE)-labeled spleen cells were transferred from donor Thy 1.2 mice to tumor-bearing host Thy 1.1 animals. Host animals were sacrificed 5 days later and the number and proliferation of Thy $1.2 \mathrm{CD}^{+}$and $\mathrm{CD} 8^{+} \mathrm{T}$-cells in the peritoneum were analyzed by flow cytometry. For some adoptive transfer experiments, inhibitory cells were added 1:1 to the host. Inhibitory cells were obtained from spleens of mice 5 days following treatment of i.p. tumors with rVVS and anti-CTLA4 but not with CTX (19).

In vitro cytotoxicity and proliferation assays. Tumor cells were plated at $1 \times 10^{4}$ cells per well of a 24 -well plate in $1 \mathrm{ml}$ in Dulbecco's modified Eagle's medium with $10 \%$ fetal calf serum.. The next day, $1 \mathrm{ml}$ with $2 \times 10^{6}$ spleen cells with 40 units $/ \mathrm{ml}$ of recombinant interleukin-2 (Teceleukin, NCI Biological Resource Branch, Frederick, MD, USA) were added to each well and plates were incubated at $37^{\circ} \mathrm{C}$. The effector cells were spleen cells which had been labelled with CFSE (Vybrant CFDA SE Cell Tracer Kit, Life Technologies Corp., Carlsbad, CA, USA) following the manufacturer's instructions. On different days as noted in the results, cells in suspension were collected first by gently washing with PBS and then adherent cells were collected after adding trypsin-EDTA (Corning Life Sciences, Tewksbury, MA, USA). Adherent T-cells in the wells were confirmed to be tumor cells by anti-HER2 staining (Biolegend, San Diego, CA, USA) and by control experiments in which no tumor cells were plated. All cells were merged for flow cytometric analysis.

Flow cytometry. Spleen or peritoneal cells $\left(1 \times 10^{6}\right)$ were suspended in ice-cold PBS $/ 0.1 \%$ bovine serum albumin $/ 0.2 \%$ azide and stained with combinations of the following antibodies: CD4allophycocyanin-eFluor 780 (eBioscience San Diego, CA, USA), CD8a- phycoerythrin (PE)-cyanine7 (eBioscience), CD90.2-PE (Becton Dickinson, Mountainview, CA, USA), CD44-BV 421 (Becton Dickinson), CD62L-BV 510 (Becton Dickinson), CD122PE (eBioscience), CD127-PE (eBioscience), CD69-PE-cyanine 7 (eBioscience), CD340-PE (erbB2/Her-2; Biolegend), Live-Dead Fixable Red Kit (ThermoFisher Scientific, Pittsburgh, PA, USA ). The following class 1 tetramers were obtained from the National Institute of Health Tetramer Core Facility at Emory University, Atlanta, GA, USA: H-2K(d)/TYLPTNASL, human HER2 p63; H2K(d)/PYVSRLLGI, human HER2 p780; H-2L(d)/MPYLIDFGL VSV-N; H-2K(d)/KYIHSANVL extracellular signal-regulated kinase-2 (ERK2) negative control. All were conjugated with Alexa 647 and used at a 1:200 dilution. Immunofluorescence was quantified using a LSR Fortesa (Becton Dickinson) and cell sorting was performed using FACSAria II (Becton Dickinson).

Statistical analysis. The log-rank statistic was used to compare survival among the treatment groups. An unpaired two-tailed $t$-test was used for all other statistical comparisons. PRISM software was used to analyze the data (GraphPad Software, Inc., La Jolla, CA, USA). 
A

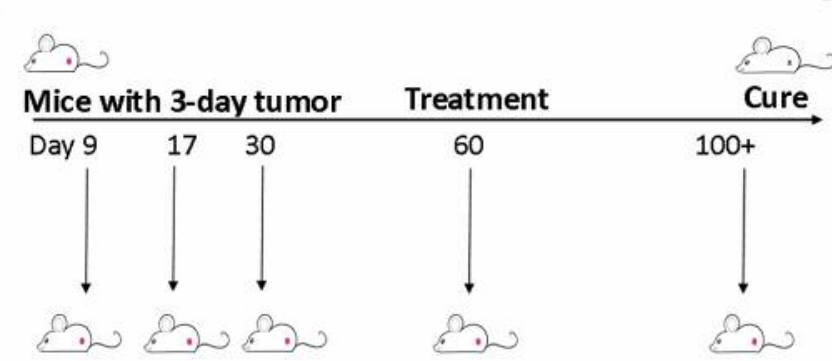

Host mice with 3-day tumor

C

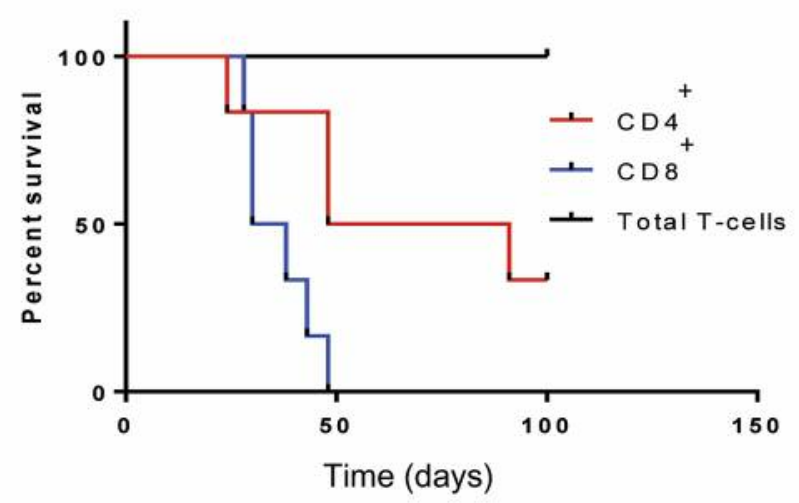

B
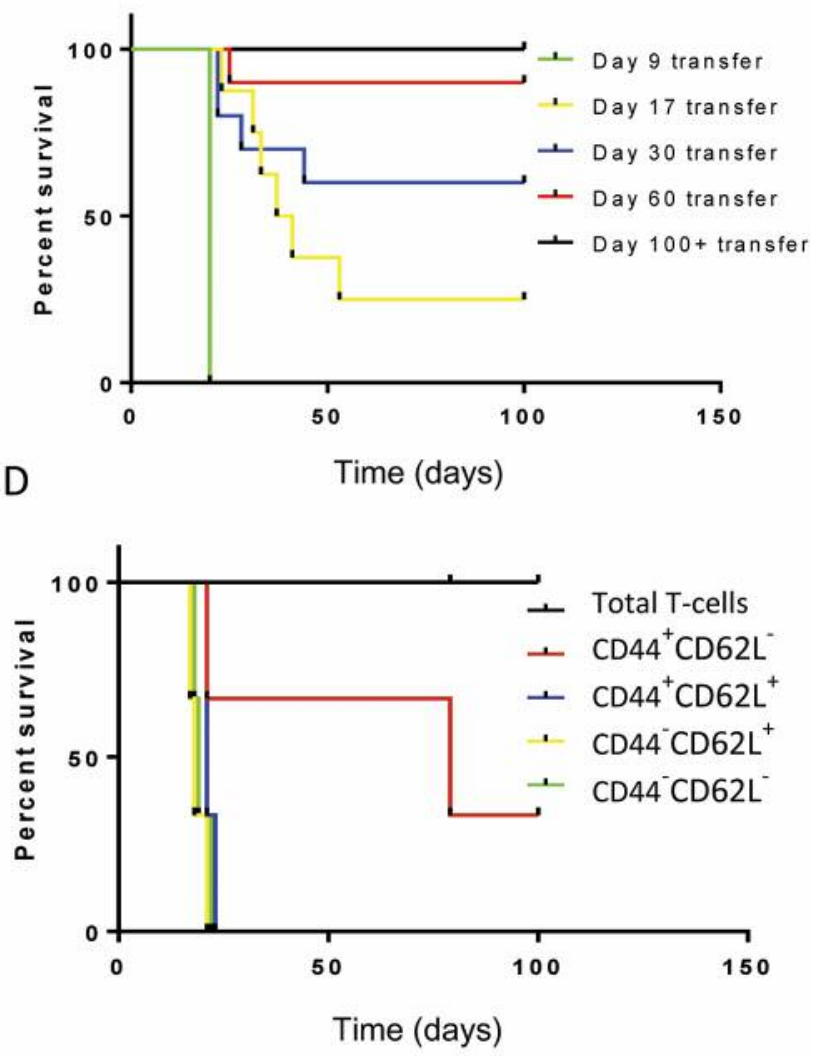

Figure 1. Survival following treatment of peritoneal tumors with T-cells from treated donors. A: Experimental paradigm. Mice implanted for 3 days with peritoneal tumor were treated with standard immuno-oncotherapy. Donor animals were sacrificed at various times after treatment and spleen cells or fractionated T-cells were transferred to host animals with peritoneal tumor. B: Cure in host mice increased steadily from 0 when day 9 effector T-cells were utilized to $25 \%$ using day-17 memory T-cells and $100 \%$ when memory T-cells were 100 days or older ( $n=10$ for transfer from day 30. 60 and 100+ donors, 8 from day 17 and 4 from day 9). C: Total T-cells, CD4+ T-cells and CD8 ${ }^{+}$T-cells from cured animals $>100$ days

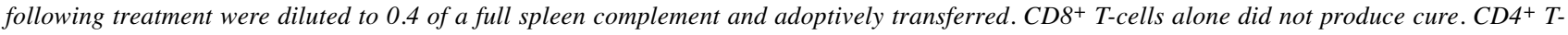
cells produced cure in 33\% and, as expected, total T-cells produced 100\% cure ( $n=6$ each for transfer of CD4 and CD8 T-cells and 5 for transfer of total T-cells). D: T-cells were fractionated into four populations based on expression of CD44 and CD62L and adoptively transferred. As expected, total T-cells produced cure but $\mathrm{CD}_{4} 4^{+}, \mathrm{CD} 62 \mathrm{~L}^{+} \mathrm{T}$-cells, a putative stem-like T-cell memory population, had no efficacy ( $n=3$ each for each group).

\section{Results}

Cure was achieved with viral onco-immunotherapy within 30 days. In the first experiment of this study, 10 mice were implanted with peritoneal tumor and treated with curative immuno-oncotherapy. $\mathrm{CD}^{+}$and $\mathrm{CD}^{+} \mathrm{T}$-cells were depleted beginning 30 days following treatment and continuing for 4 weeks. None of these animals developed tumors. This indicated tumor eradication because we have previously shown in this model that complete tumor elimination is dependent on host $\mathrm{CD}^{+}{ }^{+}$and $\mathrm{CD} 8^{+}$T-cells: depleting either of these cell types 10 days following treatment resulted in death from tumor in all animals (26). In the present study, all T-cells were depleted at 30 days and tumor did not grow, indicating that cure had been achieved. This conclusion is supported by our previous histological studies which showed that at 14 days following treatment, tumor nodules were almost entirely replaced by lipid-laden histiocytes and smaller collections of macrophages, lymphocytes and eosinophils (26).

Antitumor T-cell memory was present 30 days after viral oncoimmunotherapy. In the second experiment of this study, five animals receiving curative therapy for peritoneal neoplastic implants were re-challenged in the peritoneum 30 days following treatment with implants of the same neoplastic cell line. None of the animals developed tumor, indicating that a protective T-cell response was already present at this early time.

Cure by passive transfer of antitumor T-cell memory improved as memory matures from 30 to 100 days. Donor animals were implanted with peritoneal tumor and treated with curative immuno-oncotherapy. At different times 
A

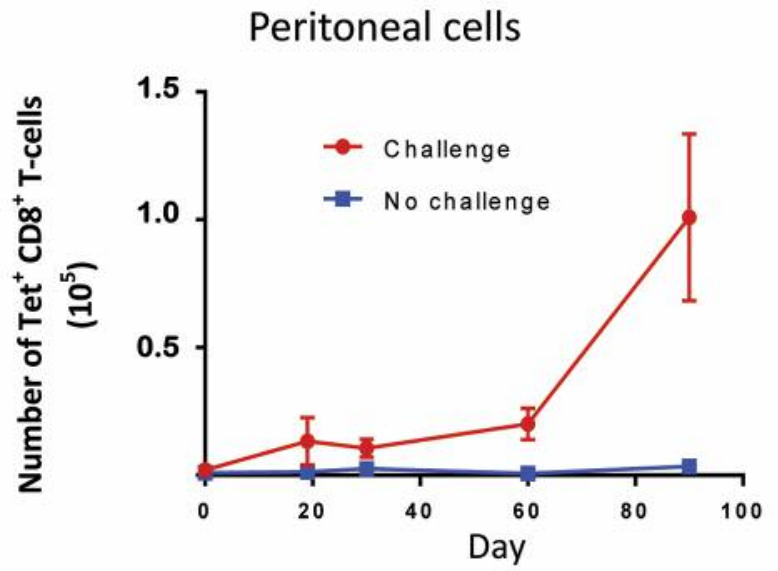

B

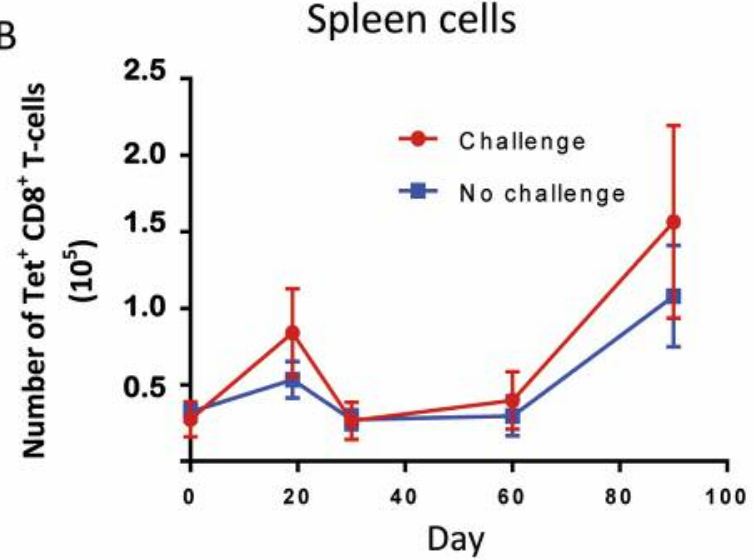

C

Peritoneal cells

Day 0

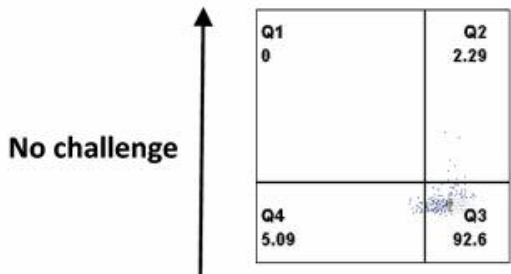

Challenge
Day 19
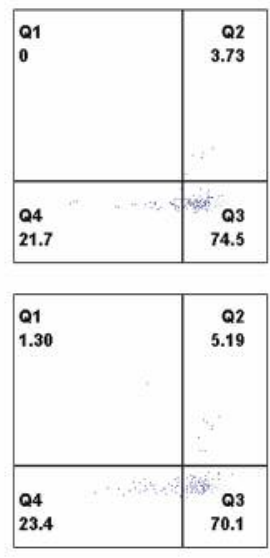

Day 30
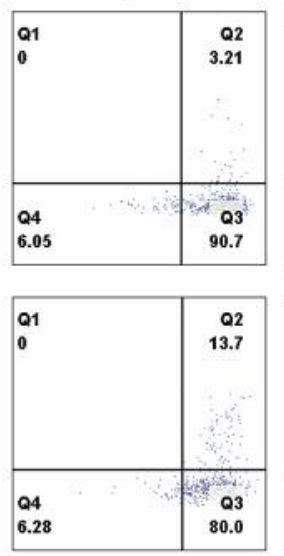

Day 60
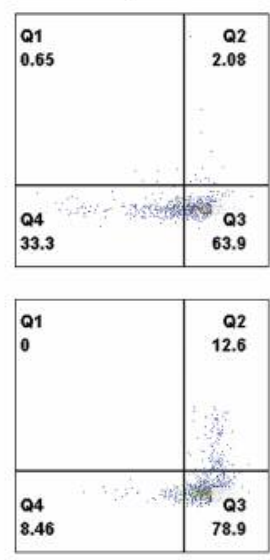

Day $100+$
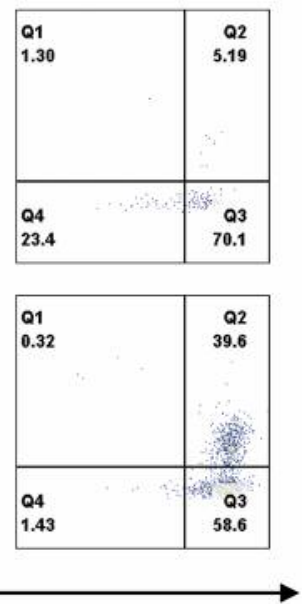

Figure 2. Tumor-specific CD8 ${ }^{+}$T-cell response to peritoneal tumor challenge in previously treated animals. A. Tumor-specific tetramer-positive (Tet+) $C D 8^{+} T$-cells in the peritoneum in animals challenged at different times following treatment ( $n=6$ or greater for each data point except one with 5; mean with SEM bars; error bars do not appear when smaller than symbols; $p=0.05$ comparing day $100+$ with naïve and $p=0.04$ comparing day 100+ with day 30 in challenged animals). B: Tumor-specific tetramer-positive CD8+ T-cells in the spleen in animals challenged at different times following treatment ( $n=6$ or greater for each data point except one with 5; mean with SEM bars; error bars do not appear when smaller than symbols; $p=0.03$ comparing day 100+ with naïve in non-challenged animals). C: Representative flow cytometric data from peritoneal cells.

following treatment the mice were sacrificed and donor Tcells were used to treat 3-day peritoneal tumors in host animals (Figure 1A). As shown in Figure 1B, cure in host mice increased steadily from $25 \%$ when 17 -day memory Tcells were utilized to $100 \%$ when memory T-cells were 100 days or older. Effector T-cells from donors 9 days following oncotherapy were completely ineffective. The $100 \%$ cure rate was achieved using T-cells fractionated from total spleen and diluted to 0.25 of a full spleen complement in five animals and 0.1 in five animals. Clearly, T-cells were the curative cells and were abundant at 100 days.
In a separate experiment, total T-cells, CD $4^{+} \mathrm{T}$-cells and $\mathrm{CD}^{+}$T-cells from cured animals $>100$ days following treatment were diluted to 0.4 of a full spleen complement and adoptively transferred to treat 3-day peritoneal tumors in host animals. $\mathrm{CD}^{+} \mathrm{T}$-cells alone did not produce cure. $\mathrm{CD}^{+}{ }^{+} \mathrm{T}$-cells produced cure in $33 \%$ and, as expected, total T-cells produced $100 \%$ cure (Figure 1C).

Unlike published work with anti-virus T-cell memory, we were not able to show that $\mathrm{CD} 44^{+}, \mathrm{CD}_{2} \mathrm{~L}^{+} \mathrm{T}$-cells identified a subpopulation of stem-like T-cell memory cells that could be transferred to tumor-bearing animals and reconstitute to curative antitumor T-cell memory (Figure 1D) (27). This 
A

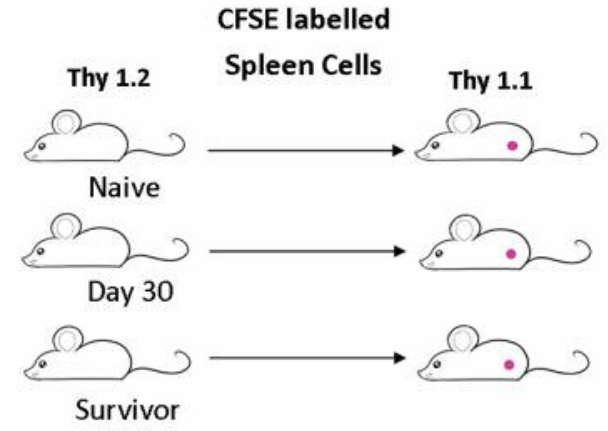

C

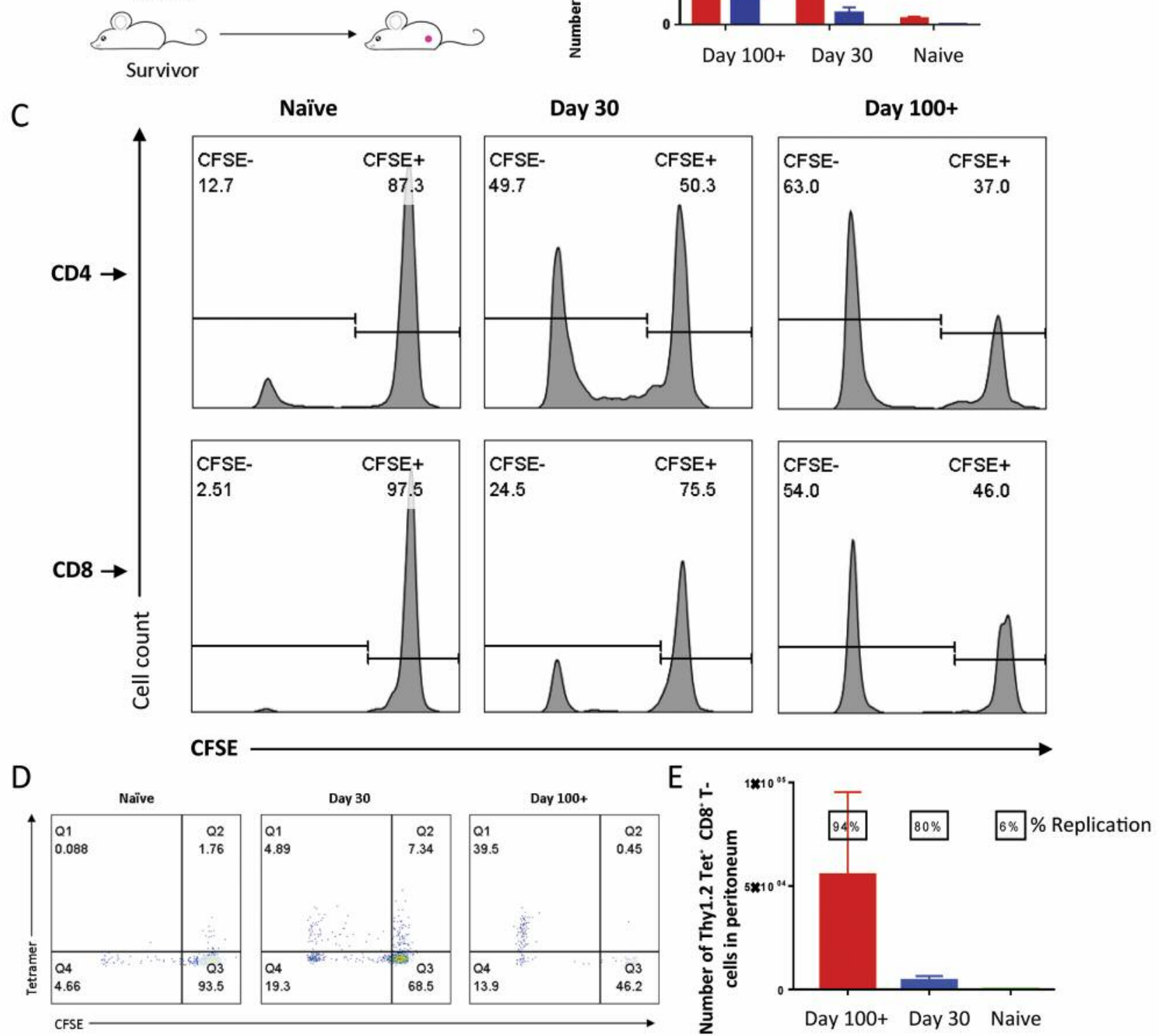

B

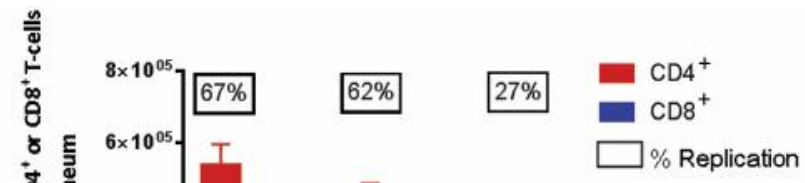

$3 \%$

Figure 3. Proliferation of $\mathrm{CD}^{+}$and $\mathrm{CD}^{+}$antitumor memory T-cells in response to tumor in vivo A: Experimental paradigm. Carboxyfluorescein succinimidyl ester (CFSE)-labeled spleen cells were transferred from donor Thy 1.2 animals into host Thy 1.1 mice with 3-day-old peritoneal tumor implants. Peritoneal cells were harvested by lavage 5 days later. Donors had undergone treatment with immuno-oncotherapy 100+ days previously, 30 days previously or were naïve animals. B: The total number of $\mathrm{CD}^{+}$and $C D 8^{+}$donor Thy 1.2 cells and the percentage with low CFSE, indicating replication, was determined by flow cytometry ( $n=6$ for each data point except 2 with 5; mean with SEM bars; $p=0.0001$ comparing number of donor CD4 T-cells from day 100+ with nä̈ve animals; $p=0.03$ comparing number of donor CD8 T-cells from day 100+ with naüve animals). C: Representative CFSE flow cytometric data from Thy $1.2 C D 4^{+}$and $C D 8^{+} T$-cells in the peritoneal washings. D: Representative CFSE flow cytometric data from tetramer-positive $\left(\mathrm{Tet}^{+}\right)$Thy $1.2 \mathrm{CD} 8^{+}$T-cells in the peritoneal washings. E. The total number of tetramer-positive CD8 ${ }^{+}$donor Thy 1.2 cells and the percentage with low CFSE, indicating replication, was determined by flow cytometry ( $n=6$ for each data point except 1 with 5 ; mean with SEM bars; differences did not reach statistical significance). 

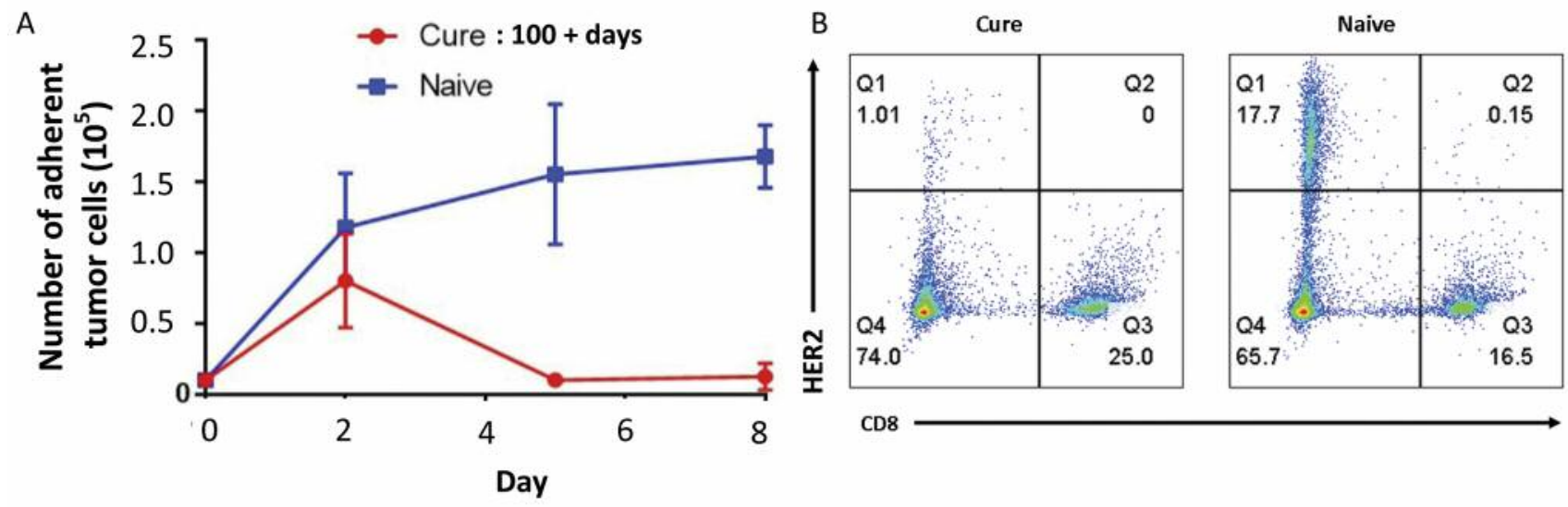

C
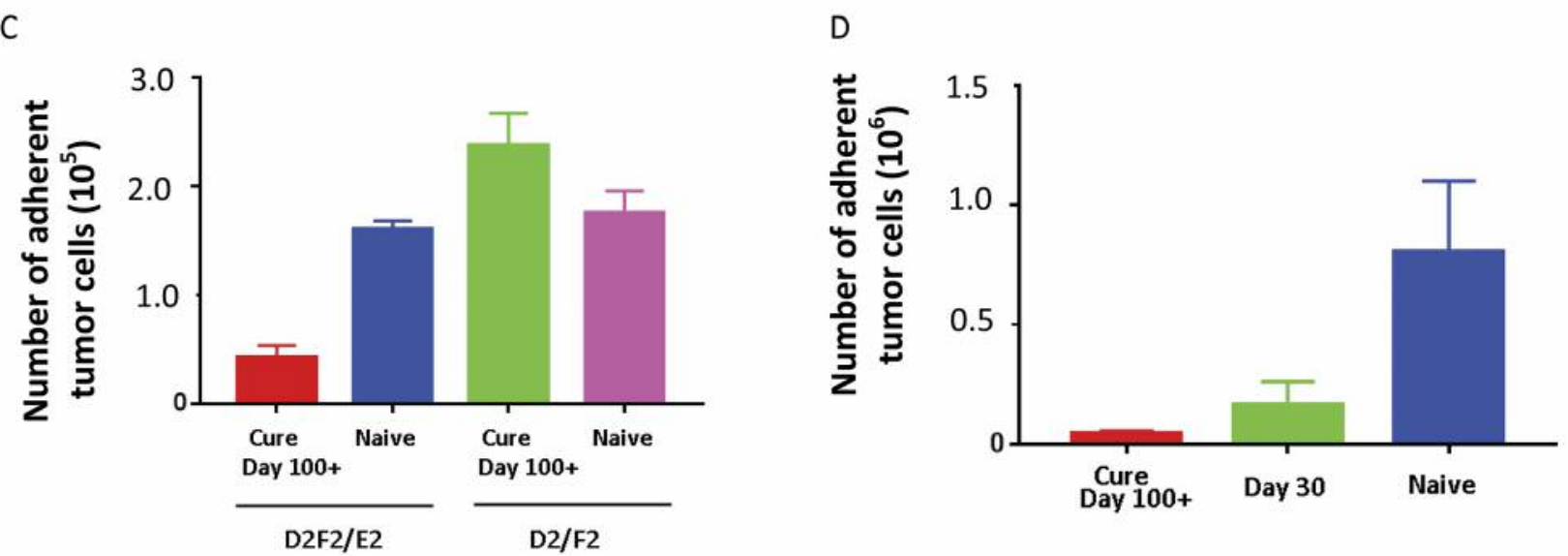

Figure 4. In vitro growth assay of tumor cells in the presence of memory T-cells. A: Tumor cells grew steadily in the presence of naïve T-cells but were killed, and tumor growth was inhibited significantly by day 100+ antitumor memory T-cells within 2 days of culture ( $n=4$ for each data point; mean with SEM bars; error bars do not appear when smaller than symbols; $p=0.026$ on day 5 and $p=0.0007$ on day 8). B: Representative flow cytometric data showing loss of human epidermal growth factor receptor 2 (HER2/neu) expressing cells in wells containing antitumor memory Tcells. C: The immuno-dominant antitumor T-cell is targeted to a HER2/neu receptor antigen and tumor cell killing is shown to be specific for $D 2 F 2 / E 2$ cells which express HER2/neu and not D2F2 cells, the parental cell line, which does not ( $n=5$ for each data point; mean with SEM bars; $p<0.0001$ comparing number of D2F2/E2 cells when incubated with antitumor memory or naïve T-cells). D: Day 30 antitumor memory T-cells had intermediate ability to inhibit growth compared with day 100+ memory and naïve T-cells ( $n=4$ for each data point; mean with/SEM bars; $p=0.04$ comparing day 100+ memory T-cells to nä̈ve T-cells).

subpopulation alone had no activity against 3-day peritoneal tumors in host animals when transferred at 1.0 spleen equivalents from cured animals $>100$ days following treatment. Interactions between different cell populations were necessary to produce cure. The only subset with some antitumor activity was $\mathrm{CD} 44^{+} \mathrm{CD}_{2} \mathrm{~L}^{-}$, similar to results reported recently in a different mammary cancer model (28).

The specific antitumor memory CD8 T-cell response increased from 30 to 100 days after viral onco-immunotherapy. The specific antitumor memory $\mathrm{CD} 8^{+} \mathrm{T}$-cell response in the peritoneum and spleen was quantified by flow cytometric analyses using a tetramer displaying the immunodominant $\mathrm{p} 63$ epitope of the HER2/neu receptor protein. At various time following treatment of peritoneal tumors, mice were challenged once with i.p. tumor cells and sacrificed 5 days later with harvest of peritoneal lavage and spleen cells. The response to tumor challenge increased dramatically in animals surviving for 100 days after viral oncotherapy (Figure 2A and B). The number of tetramer-positive cells in the peritoneum was 10-fold higher in animals $100+$ days after oncotherapy compared with animals 30 days from oncotherapy $(p=0.04)$. There was a 50 -fold difference when comparing $100+$ day animals with naïve mice $(p=0.05)$. Illustrative flow cytometric graphs from individual animals are shown in Figure 2C. The baseline mean number of tetramer-positive cells in naïve animals was $3.2 \times 10^{4}$. This represents the false positive staining rate for this tetramer because the number of naïve $\mathrm{T}$ - 

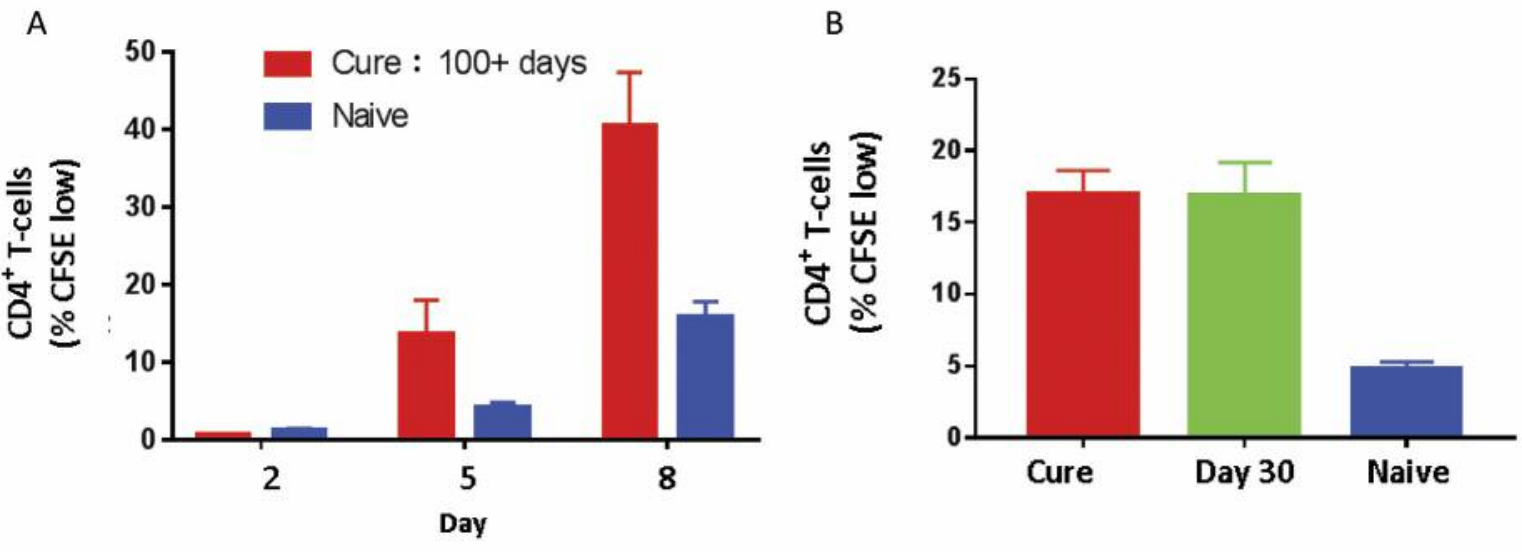

C

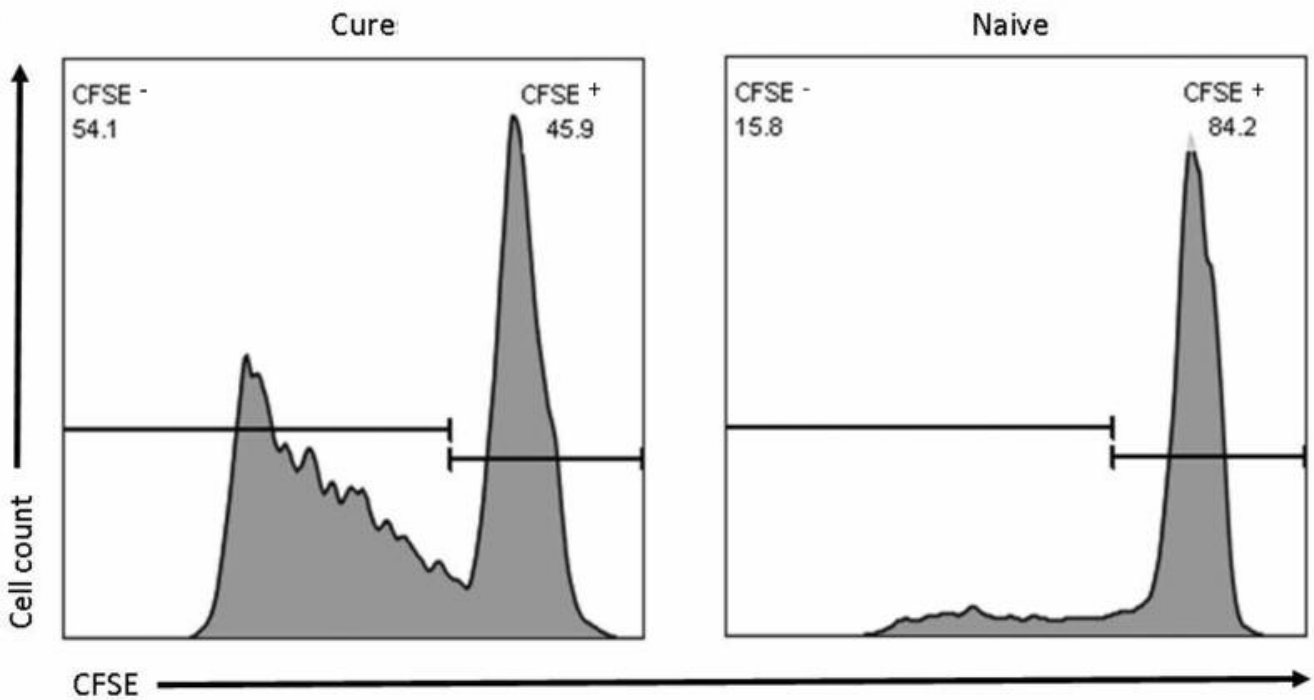

Figure 5. In vitro proliferation assay of antitumor memory CD4+ T-cells in the presence of tumor cells. A: Proliferation was assayed by loss of carboxyfluorescein succinimidyl ester (CFSE) over time. Spleen cells were labeled with CFSE prior to incubation with tumor cells and CFSE was quantified per cell by flow cytometry on different days. Proliferation of memory CD4 ${ }^{+} T$-cells was clear by day 5 and was significantly higher than that of naïve $\mathrm{CD}^{+}{ }^{+} T$-cells on day 8 ( $p=0.008 ; n=6$ or 7 for each data point; mean with SEM bars). B: Day-30 memory CD4 ${ }^{+} T$-cells were compared with day 100+ memory $\mathrm{CD}^{+}$and naïve $\mathrm{CD}^{+}$T-cells on day 5 of incubation. Proliferation was nearly identical for the two ages of memory $\mathrm{CD} 4^{+}$ $T$-cells and both were significantly greater than that of naïve T-cells ( $p=0.0003$ for naïve vs. day 100+ and $p=0.002$ for nä̈ve vs. day 30; $n=4$ for each data point; mean with SEM bars). C: Flow cytometric graphs show clear laddering of CFSE staining in the proliferating memory CD4 ${ }^{+}$ T-cells that was not seen in the naïve cells, which showed only the expected non-specific loss of CFSE over time.

cells to any single epitope in the mouse is only 15-1,000 (29). The mean number of tetramer-positive cells in cured day $100+$ mice was $1.1 \times 10^{5}$, a 3.4 -fold increase (Figure $2 \mathrm{~B} ; p=0.03$ ). The difference, $7.9 \times 10^{4}$, is a rough estimate of the steady-state number of immunodominant antitumor $\mathrm{CD}^{+}$T-cells following cure with oncotherapy. The variability of results when challenging day $100+$ mice was large probably because some animals responded to a different class I epitope than p63 and these produced a much lower number of p63 tetramerpositive $\mathrm{CD} 8^{+} \mathrm{T}$-cells than the animals responding to the usual p63 immunodominant epitope.
Specificity was determined by comparing cured animals challenged with the primary mouse tumor cell line containing the transfected HER2/neu gene, D2F2/E2 with animals challenged with the native mouse cell line, D2F2. The number of tetramer-positive cells in the peritoneum was 10-fold higher in the animals challenged with the HER2/neu expressing cells, $1.45 \times 10^{5}$ compared with $1.42 \times 10^{4}$. Pairs of mice were challenged on the same day and sacrifice and analyses occurred on the same day ( $n=2$ pairs).

Tetramer-positive $\mathrm{CD}^{+}{ }^{+} \mathrm{T}$-cells were compared in mice surviving 100 and 200 days following oncotherapy. All mice 
A

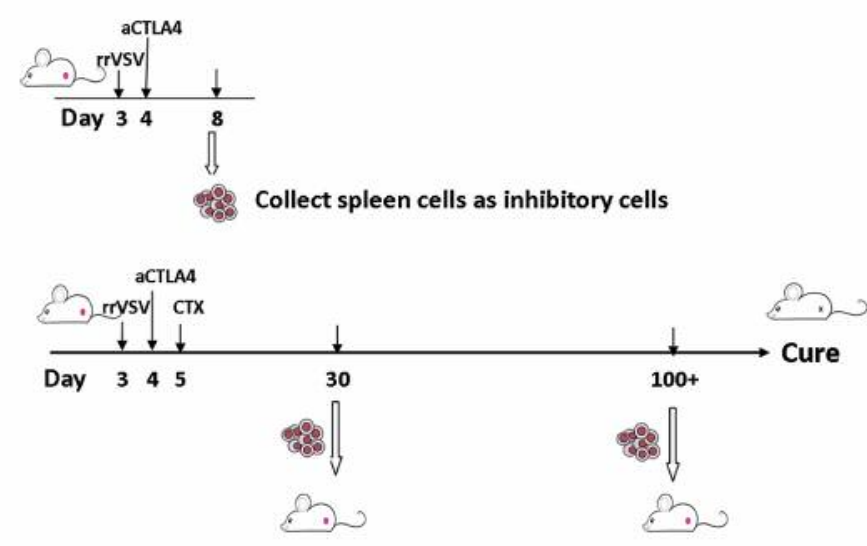

B

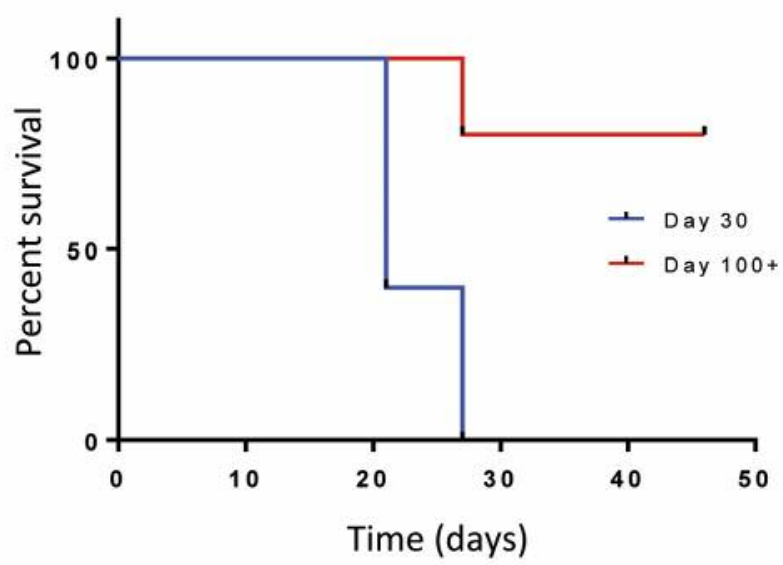

Figure 6. Mature antitumor memory T-cells were not inhibited by suppressor cells, but immature memory cells were. A: Experimental paradigm. Inhibitory cells were obtained from mice implanted with peritoneal tumor and treated with replicating recombinant vesicular stomatitis virus (rrVSV) and anti-cytotoxic T-lymphocyte-associated protein 4 (CTLA4) but not cyclophosphamide (CTX). Spleens were harvested 5 days after virus administration and total spleen cells were used for inhibition. B: Survival curves of mice with tumor implants treated with transferred memory cells with or without transferred suppressor cells. Day-30 memory T-cells were very sensitive to inhibition, whereas day 100+ memory they were resistant ( $n=5$ each for each group; $p=0.008)$.

( $\mathrm{n}=3$ in each group) were challenged on the same day and sacrifice and analyses occurred on the same day. The number of tetramer-positive T-cells was nearly identical in both groups. In the peritoneum, there were $5.32 \times 10^{4}$ in day 100 animals and $5.05 \times 10^{4}$ in day 200 animals. In the spleen there were $4.03 \times 10^{4}$ in day 100 animals and $4.73 \times 10^{4}$ in day 200 animals. These results imply that the memory response was fully mature by day 100 . We analyzed the cell surface markers CD44, CD62L, CD122, CD127 and CD69 but could not determine a pattern that distinguished effector T-cells from memory T-cells. These results were consistent with our inability to use these markers to find a subpopulation that mediated cure by adoptive transfer as reported above.

Day 100 antitumor memory CD4 and CD8 T-cells increased more rapidly in vivo in response to tumor than day 30 memory T-cells. Specific identification of an antitumor T-cell response was limited in our system to a single CD8 epitope. In order to detect the CD4 response and the full breadth of the CD8 response, we enriched the yield of antitumor T-cells using congenic animals. Spleen cells were transferred from donor Thy 1.2 animals that had undergone treatment with immunooncotherapy for i.p. tumor into host Thy 1.1 mice that were implanted i.p. with the same tumor cells (Figure 3A). Peritoneal cells were harvested by lavage 5 days later. Figure 3B shows that the number of $\mathrm{CD}^{+}$and $\mathrm{CD} 8^{+}$transferred Thy $1.2 \mathrm{~T}$-cells was significantly greater in host animals receiving adoptive transfer from donors cured for $100+$ days compared with naïve donors. The number of $\mathrm{CD}^{+}$and $\mathrm{CD} 8^{+} \mathrm{T}$-cells in recipients from 30 - day donors was intermediate. Correspondingly, proliferation of donor Thy $1.2 \mathrm{CD}^{+}$and $\mathrm{CD}^{+}$T-cells, assessed by loss of CFSE staining, was greater in the 100+- and 30-day donors compared with the naïve donors (Figure 3B). Illustrative flow cytometry graphs from individual animals are shown in Figure 3C. These results were corroborated, and indeed shown more clearly, by tetramer analysis, which found almost no tetramerpositive $\mathrm{CD}^{+} \mathrm{T}$-cells in hosts receiving naïve donor spleen cells and increasing numbers of tetramer-positive T-cells in hosts receiving cells from 30-day and 100+-day donors (Figure 3D). Correspondingly, proliferation was a minimal $6 \%$ in the few tetramer-positive cells from naïve animals compared with $80 \%$ in the 30-day donors and $94 \%$ in the 100+-day donors (Figure 3E) As expected, the number of tetramer-positive cells was a smaller subset of the larger total $\mathrm{CD} 8^{+} \mathrm{T}-$ cell response.

In vitro assays detected antitumor memory T-cells by tumor cell killing and $\mathrm{CD}^{+}{ }^{+}$-cell proliferation. In vitro growth and proliferation assays were also able to detect memory antitumor T-cell responses without using tetramers. Figure $4 \mathrm{~A}$ shows that the D2F2/E2 tumor cell line grew steadily in the presence of naïve T-cells but tumor cells were killed and growth inhibited significantly by day $100+$ antitumor memory T-cells within 2 days of culture ( $p=0.026$ on day 5 and $p=0.0007$ on day 8 ). Flow cytometry confirmed the growth inhibition in all cases (Figure 4B). Growth inhibition depended on the presence of the HER2/neu receptor because, in a separate experiment, the transfected cell line, D2F2/E2, was significantly inhibited by 100+-day antitumor memory T-cells compared to naïve T-cells $(p<0.0001)$ but these same memory cells did not inhibit 
growth of the parent cell line, D2F2, which did not express the receptor (Figure 4C). Anti-virus memory T-cells created in mice by infection with wild-type VSV 3 months previously also did not inhibit tumor cell growth, behaving just like naïve T-cells (data not shown). Day 30 antitumor memory T-cells had intermediate ability to inhibit growth compared with day 100+ memory and naïve T-cells (Figure 4D).

Proliferation was assayed by loss of CFSE over time. Spleen cells were labeled with CFSE prior to incubation with tumor cells and CFSE was quantified per cell by flow cytometry on various days. Proliferation of memory CD4 Tcells was clear by day 5 and was significantly higher than naïve CD4 T-cells on day 8 (Figure $5 \mathrm{~A} ; p=0.008$ ). Review of the flow cytometric graphs showed clear laddering of CFSE staining in the proliferating memory $\mathrm{CD} 4^{+} \mathrm{T}$-cells that was not seen in the naïve cells, which showed only the expected nonspecific loss of CFSE over time (Figure 5C). In a separate experiment, day 30 memory $\mathrm{CD}^{+} \mathrm{T}$-cells were compared with day $100+$ memory $\mathrm{CD}^{+}$and naïve $\mathrm{CD} 4^{+} \mathrm{T}$-cells on day 5 of incubation. Proliferation was nearly identical for the two ages of memory $\mathrm{CD} 4^{+} \mathrm{T}$-cells and both were significantly greater than naïve T-cells (Figure $5 \mathrm{~B} ; p=0.0003$ for naïve vs. day $100+$ and $p=0.002$ for naïve vs. day 30). Our flow cytometry-based assay did not demonstrate specific CD8 Tcell proliferation in part, we think, because antitumor $\mathrm{CD}^{+}$ T-cells died during incubation with tumor cells and nonspecific staining of these dead cells precluded further analyses.

Day 100+ antitumor memory cells were resistant to suppressor cells but day 30 memory cells were not. Our previous work has shown that memory antitumor T-cells resist inhibition by immune suppressor cells (19). The same inhibitory cells that were able to abrogate an acute therapeutic T-cell response to established tumor did not inhibit a therapeutic response produced by memory T-cells. Inhibitory cells were obtained from mice implanted with peritoneal tumor and treated with rrVSV and anti-CTLA4 but not CTX. Spleens were harvested 5 days after virus administration and total spleen cells were used for inhibition. Our previous work showed that T-cells alone or non-T-cells alone (presumably macrophage-derived suppressor cells) were fully inhibitory, indicating that the total inhibitory effect was pleomorphic and redundant. Here we used the same paradigm of inhibition (Figure 6A) to show that day 30 memory T-cells were very sensitive to inhibition, whereas day $100+$ memory were resistant (Figure 6B; $p=0.008$ ). The difference between the survival curves was highly significant statistically but the interpretation that day $100+$ memory is more resistant to inhibition than that at day 30 is qualitative because day 30 memory T-cells without inhibitors yielded $60 \%$ cure rate compared with $100 \%$ for day $100+$ memory T-cells. Still, the absence of any resistance to inhibition by day 30 memory cells suggests that day 100+ memory cells have developed new functional properties. Fully mature antitumor memory T-cells are therefore likely to be more effective in preventing latedeveloping metastases than partially mature memory.

\section{Discussion}

The major finding in this study is that therapeutically potent antitumor memory is generated by immuno-oncotherapy 30 days after treatment and matures to maximal potency at 100 days. Maturation was not uniform across different measures. The results are relevant to humans because a similar timecourse of non-uniform maturation was found following human vaccination for yellow fever and smallpox (11). Day 30 memory was completely effective in preventing tumor rechallenge at the primary site but was less effective than day $100+$ memory in curing tumor by adoptive therapy into different host animals. Day $100+$ memory T-cells were superior to day 30 cells in responding to tumor challenge in vivo and in killing assays in vitro but not in proliferation assays in vitro. In one critical measure, day 30 memory cells were markedly inferior to day $100+$ cells: day 30 cells were completely inhibited by suppressor cells and day 100+ memory cells were not. This study did not determine the mechanisms of the differential response and future work must determine not only molecular, cellular, genetic and epigenetic mechanisms but such basic questions as number, location and functional characteristics of the memory T-cells at different times. The current study did suggest that the number of resting antitumor memory T-cells increased in the spleen from 30 to 100 days and that day $100+$ memory T-cells developed new functional capabilities. No matter the mechanism involved, it is clear that rrVSV, anti-CTLA4 and CTX are potent stimulators of antitumor memory T-cells and these cells require 100 days to reach their highest functionality in a mouse model system that has no ongoing exposure to tumorderived antigen because the tumor has been cured (8). The surest method then of generating these powerful antitumor memory T-cells in the clinic will be to pair vaccination at initial presentation with eradication of all active tumor for at least several months. Powerful memory T-cells are then available to survey the body and eliminate recurrence or spread from latent sites at a very early stage just as viral memory T-cells do for herpes simplex virus, varicella zoster virus, Epstein Barr virus and papovavirus. These latent metastatic sites could be in known sanctuary sites such as brain or testis, or even in lymph nodes, lymph organs or the lung if small cell collections do not provoke an inflammatory response. Vaccination in the presence of chronic active tumor is likely to be ineffective as it is with chronic clinical infections such as tuberculosis, malaria and HIV and in a mouse model system of lymphocytic choriomeningitis virus $(30,31)$. Another important implication of this work is that multiple closely spaced immunizations may be a poor strategy 
for evoking a powerful mature T-cell response, which requires prolonged antigen freedom in order to evolve (32-34).

In this work, as well as our previous studies, we found that adoptive transfer of memory $\mathrm{CD}^{+}{ }^{+}$T-cells alone can sometimes cure tumor in host animals, that transfer of memory $\mathrm{CD}^{+} \mathrm{T}$-cells alone rarely does so, and that the combination of memory $\mathrm{CD}^{+}$and $\mathrm{CD}^{+}$T-cells is remarkably potent. $\mathrm{CD} 4^{+} \mathrm{T}$-cells are known to stimulate $\mathrm{CD} 8^{+} \mathrm{T}$-cells and to recruit and activate various immune cells to target infection and neoplasia. In addition, $\mathrm{CD}^{+}{ }^{+} \mathrm{T}$-cells may themselves sometimes have the ability to kill cancer cells (35). In this model system, $\mathrm{CD}^{+} \mathrm{T}$-cells are dependent on $\mathrm{CD} 4^{+} \mathrm{T}$-cells for therapeutic effect but in other systems including the use of chimeric antigen receptor T-cells in humans, they may be therapeutic by themselves (8). Future work on mechanisms must, therefore, study not only intracellular molecular maturation but also time-dependent changes in intercellular communication.

Importantly, this work was carried out in mice with a normal diversity of T-cells and should therefore be directly relevant to the clinical situation $(8,36)$. The findings are supported by functional assays, which are direct measures of activity, as opposed to surface markers, which are indirect and often imprecise, with broad patterns of expression which vary under different experimental conditions $(8,11,36,37)$. We tested CD44, CD62L, CD122, CD127 and CD69 in all of our flow cytometric assays and were not able to find a pattern that clearly and consistently distinguished memory $\mathrm{CD}^{+}{ }^{+}$or $\mathrm{CD}^{+}$ T-cells from effector T-cells. We were therefore unable to expose mixed T-cells to tumor antigen and distinguish a naïve response from a memory response, a problem also encountered by others (36). Our functional assay did not support CD44 ${ }^{\text {hi }}$,

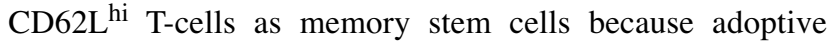
transfer of this cell population had no therapeutic efficacy. Differences in model system may explain why our results differ from other investigators. We treated cancer not infection, using Balb/c and not C57/B16 mice and did not use mice with a monoclonal T-cell repertoire (27). A robust general marker of T-cell memory would greatly improve the signal-to-noise problem inherent in assaying a mixed T-cell population. The number of tumor-specific memory T-cells is a small fraction of the total T-cell population. We found that the steady-state number of immunodominant antitumor memory $\mathrm{CD}^{+} \mathrm{T}$-cells following cure with oncotherapy was roughly $8 \times 10^{4}$ cells out of a total of $\sim 1 \times 10^{8}$ spleen cells, a number consistent with a human study of anti-viral memory T-cells (11). Our in vivo studies required a CD8 tetramer to the immunodominant epitope or enrichment using transfer between congenic mice to yield analyzable results and even here, use of the tetramer showed a much cleaner picture than use of congenic animals (compare Figure 3A and B). Every T-cell assay has potential for significant false-positive and false-negative results, except for those using tetramer (11). However, comprehensive tetramers are not usually available for clinical studies. Further study will be required to determine which of our assays correlates with the clinically relevant outcome of protection from latent metastases and then how to optimally generate the greatest quantity of the most powerful memory T-cells. Generating antitumor memory T-cells may be a highly potent strategy against cancer with late-developing metastases.

\section{Conflicts of Interest}

IB: US Patent Application 7,429,481 entitled "Targeting viruses using a modified Sindbis glycoprotein" made September 30. 2008. The targeted virus is tested in this animal work. IB has no affiliation with any company.

\section{Conflicts of Interest}

YG and MAB have no conflict of interest to report.

\section{Funding}

US Army Medical Research Breast Cancer IDEA Award (BC101672) to IB.

\section{Acknowledgements}

The Authors thank Drs. Wei-Zen Wei, John K. Rose, Irvin S. Y. Chen and Genentech Inc. who very generously supplied materials as noted in the text. We thank Joshua Michel, Senior Technician and Flow Core Manager for invaluable technical assistance with Flow Cytometry.

The Authors gratefully acknowledge the assistance of the NIH Tetramer Core Facility at Emory University and Dale Long, facility manager, for generously supplying all the tetramer reagents. The NIH Tetramer Facility is supported by contract HHSN272201 300006C from the National Institute of Allergy and Infectious Diseases, a component of the National Institutes of Health in the Department of Health and Human Services.

\section{References}

1 Novitzky N and Rouskova A: Infectious complications following T-cell depleted hematopoietic stem-cell transplantation. Cytotherapy 3: 165-173, 2001.

2 Pachlopnik Schmid J, Gungor $\mathrm{T}$ and Seger R: Modern management of primary T-cell immunodeficiencies. Pediatr Allergy Immunol 25: 300-313, 2014.

3 Moon JJ and Jenkins MK: The human T-cell repertoire grows up. Immunol Cell Biol 93: 601-602, 2015.

4 Bingaman AW and Farber DL: Memory T-cells in transplantation: generation, function and potential role in rejection. Am J Transplant 4: 846-852, 2004.

5 Zediak VP, Wherry EJ and Berger SL: The contribution of epigenetic memory to immunologic memory. Curr Opin Genet Dev 21: 154-159, 2011.

6 O'Sullivan D, van der Windt GJ, Huang SC, Curtis JD, Chang CH, Buck MD, Qiu J, Smith AM, Lam WY, DiPlato LM, Hsu FF, Birnbaum MJ, Pearce EJ and Pearce EL: Memory CD8(+) T-cells use cell-intrinsic lipolysis to support the metabolic programming necessary for development. Immunity 41: 75-88, 2014. 
7 Kaech SM and Cui W: Transcriptional control of effector and memory CD8+ T-cell differentiation. Nature Rev Immunol 12: 749-761, 2012.

8 Jameson SC and Masopust D: Diversity in T-cell memory: an embarrassment of riches. Immunity 31: 859-871, 2009.

9 Kaech SM, Hemby S, Kersh E and Ahmed R: Molecular and functional profiling of memory CD8 T-cell differentiation. Cell 111: 837-851, 2002.

10 Wherry EJ, Teichgraber V, Becker TC, Masopust D, Kaech SM, Antia R, von Andrian UH and Ahmed R: Lineage relationship and protective immunity of memory CD8 T-cell subsets. Nat Immunol 4: 225-234, 2003.

11 Miller JD, van der Most RG, Akondy RS, Glidewell JT, Albott S, Masopust D, Murali-Krishna K, Mahar PL, Edupuganti S, Lalor S, Germon S, Del Rio C, Mulligan MJ, Staprans SI, Altman JD, Feinberg MB and Ahmed R: Human effector and memory $\mathrm{CD}^{+}{ }^{+} \mathrm{T}$-cell responses to smallpox and yellow fever vaccines. Immunity 28: 710-722, 2008.

12 Roberts $\mathrm{AD}$, Ely $\mathrm{KH}$ and Woodland DL: Differential contributions of central and effector memory T-cells to recall responses. J Exp Med 202: 123-133, 2005.

13 Gourley TS, Wherry EJ, Masopust D and Ahmed R: Generation and maintenance of immunological memory. Semin Immunol 16: 323-333, 2004.

14 Roncarolo MG and Battaglia M: Regulatory T-cell immunotherapy for tolerance to self antigens and alloantigens in humans. Nature Rev Immunol 7: 585-598, 2007.

15 McLachlan SM and Rapoport B: Autoimmune hypothyroidism: T-cells caught in the act. Nat Med 10: 895-896, 2004.

16 Davila ML and Sadelain M: Biology and clinical application of CAR T-cells for B-cell malignancies. Int J Hematol 104: 6-17, 2016.

17 Degn M and Kornum BR: Type 1 narcolepsy: a CD8(+) T-cellmediated disease? Ann N Y Acad Sci 1351: 80-88, 2015.

18 Gao Y, Whitaker-Dowling P, Griffin JA and Bergman I: Treatment with targeted vesicular stomatitis virus generates therapeutic multifunctional antitumor memory CD4 T-cells. Cancer Gene Ther 19: 282-291, 2012.

19 Gao Y, Whitaker-Dowling P and Bergman I: Memory antitumor T-cells resist inhibition by immune suppressor cells. Anticancer Res 35: 4593-4597, 2015.

20 Gao Y, Whitaker-Dowling P, Barmada MA, Basse PH and Bergman I: Viral infection of implanted meningeal tumors induces antitumor memory T-cells to travel to the brain and eliminate established tumors. Neuro Oncol 17: 536-544, 2015.

21 Wentworth L, Meyers JV, Alam S, Russ AJ, Suresh M and Cho CS: Memory T-cells are uniquely resistant to melanoma-induced suppression. Cancer Immunol Immunother 62: 149-159, 2013.

22 Ratasvuori M, Wedin R, Hansen BH, Keller J, Trovik C, Zaikova O, Bergh P, Kalen A and Laitinen M: Prognostic role of en-bloc resection and late onset of bone metastasis in patients with bone-seeking carcinomas of the kidney, breast, lung and prostate: SSG study on 672 operated skeletal metastases. J Surg Oncol 110: 360-365, 2014

23 Friberg S and Nyström A: Cancer metastases: early dissemination and late recurrences. Cancer Growth Metastasis 8: 43-49, 2015.

24 Kaufman HL, Kohlhapp FJ and Zloza A: Oncolytic viruses: a new class of immunotherapy drugs. Nat Rev Drug Discov 14: 642-662, 2015.
25 Bergman I, Whitaker-Dowling $\mathrm{P}$, Gao $\mathrm{Y}$ and Griffin JA: Preferential targeting of vesicular stomatitis virus to breast cancer cells. Virology 330: 24-33, 2004.

26 Gao Y, Whitaker-Dowling P, Griffin JA, Barmada MA and Bergman I: Recombinant vesicular stomatitis virus targeted to HER2/neu combined with anti-CTLA4 antibody eliminates implanted mammary tumors. Cancer Gene Ther 16: 44-52, 2009.

27 Graef P, Buchholz VR, Stemberger C, Flossdorf M, Henkel L, Schiemann M, Drexler I, Hofer T, Riddell SR and Busch DH: Serial transfer of single-cell-derived immunocompetence reveals stemness of CD8(+) central memory T-cells. Immunity 41: 116-126, 2014.

28 Spitzer MH, Carmi Y, Reticker-Flynn NE, Kwek SS, Madhireddy D, Martins MM, Gherardini PF, Prestwood TR, Chabon J, Bendall SC, Fong L, Nolan GP and Engleman EG: Systemic immunity is required for effective cancer immunotherapy. Cell 168: 487-502.e415, 2017.

29 Jenkins MK and Moon JJ: The role of naive T-cell precursor frequency and recruitment in dictating immune response magnitude. J Immunol 188: 4135-4140, 2012.

30 Seder RA and Hill AV: Vaccines against intracellular infections requiring cellular immunity. Nature 406: 793-798, 2000.

31 Wherry EJ, Blattman JN, Murali-Krishna K, van der Most R and Ahmed R: Viral persistence alters CD8 T-cell immunodominance and tissue distribution and results in distinct stages of functional impairment. J Virol 77: 4911-4927, 2003.

32 Wirth TC, Xue HH, Rai D, Sabel JT, Bair T, Harty JT and Badovinac VP: Repetitive antigen stimulation induces stepwise transcriptome diversification but preserves a core signature of memory CD8(+) T-cell differentiation. Immunity 33: 128-140, 2010.

33 Fraser KA, Schenkel JM, Jameson SC, Vezys V and Masopust D: Preexisting high frequencies of memory CD8 ${ }^{+} \mathrm{T}$-cells favor rapid memory differentiation and preservation of proliferative potential upon boosting. Immunity 39: 171-183, 2013.

34 Masopust D, Ha SJ, Vezys V, Ahmed R, Masopust D, Ha SJ, Vezys V and Ahmed R: Stimulation history dictates memory CD8 T-cell phenotype: implications for prime-boost vaccination. J Immunol 177: 831-839, 2006.

35 Brown DM: Cytolytic CD4 cells: Direct mediators in infectious disease and malignancy. Cell Immunol 262: 89-95, 2010.

36 Tang AL, Bingaman AW, Kadavil EA, Leeser DB and Farber DL: Generation and functional capacity of polyclonal alloantigen-specific memory CD4 T-cells. Am J Transplant 6: 1275-1284, 2006.

37 Steinert EM, Schenkel JM, Fraser KA, Beura LK, Manlove LS, Igyarto BZ, Southern PJ and Masopust D: Quantifying memory CD8 T-cells reveals regionalization of immunosurveillance. Cell 161: 737-749, 2015. 